\title{
Decisión basada en el método mismatch DWI/FLAIR en el tratamiento endovascular con despliegue de stent Solitaire, para el accidente cerebrovascular del despertar: caso clínico
}

\section{MR Imaging-Based Decision in Endovascular Treatment with Solitaire Deployment, for Stroke on Awakening: Case Report}

\author{
Matías Negrotto ${ }^{1}$ Roberto Crosa ${ }^{2}$ Nicolas Sgarbi ${ }^{3}$ Alejandra Jaume ${ }^{3}$
}

${ }^{1}$ Hospital Maciel, Montevideo, Uruguay
${ }^{2}$ Centro Endovascular Neurológico, Montevideo, Uruguay
${ }^{3}$ Hospital de Clínicas, Montevideo, Uruguay

Rev Argent Radiol 2018;82:139-143.

Estimados Editores,

El accidente cerebrovascular (ACV) del despertar, definido como la situación en la que un paciente se despierta con síntomas de ACV que no estaban presentes antes de dormirse, representa hasta $25 \%$ de los ACV isquémicos agudos. ${ }^{1}$ Varios estudios sugieren que la mayoría de esos ACV ocurren cerca del momento de despertarse. ${ }^{2}$ Los pacientes con ACV del despertar han sido excluidos de la mayoría de los ensayos clínicos de tratamientos para el ACV isquémico ya que muchas veces no cumplen los criterios para la terapia de reperfusión aguda en la práctica clínica, lo que lleva a resultados deficientes. Los estudios de neuroimagen pueden ayudar a seleccionar esos pacientes para terapias de reperfusión aguda. Tal es el caso de los avances en estudios de resonancia magnética (RM), que han permitido una mejor caracterización del estado del tejido y los vasos en el ACV agudo. El concepto de mismatch entre la imagen ponderada en difusión (DWI) y la secuencia de recuperación de la inversión atenuada por líquido (FLAIR) estima de manera indirecta el momento del evento isquémico. ${ }^{3}$ El propósito de este trabajo es describir el caso clínico de una paciente que se sometió con éxito a un tratamiento neuroendovascular por un ACV del despertar sin complicaciones importantes y con un excelente resultado clínico, gracias a una ampliación de la ventana terapéutica guiada por RM.

Presentamos una mujer de 82 años de edad con antecedentes de hipertensión y dislipidemia que se despierta
Address for correspondence Matías Negrotto, Medical Reseident, Jaime Zudañez 2863, Hospital Maciel, Montevideo, Uruguay (e-mail: matiasnegrotto@gmail.com).

con un síndrome de ACV de la arteria cerebral media (ACM) derecha con parálisis facial izquierda, desviación de la mirada a la derecha y debilidad del lado izquierdo; un puntaje de 12 en la Escala de accidente cerebrovascular de los Institutos Nacionales de Salud de los EE.UU. (NIHSS: National Institutes of Health Stroke Scale). La RM cerebral (Signa 1.5T) realizada 75 minutos después de que la paciente se despertó (-Fig. 1), mostró leve hiperintensidad en el territorio de la ACM profunda derecha en la secuencia DWI con una disminución del coeficiente de difusión aparente (ACD). La secuencia FLAIR ponderada en T2 no mostró cambios en la intensidad de señal del parénquima. La angiografía por RM con la secuencia time of flight (TOF) en 3D mostró una oclusión proximal de la ACM derecha. La ausencia de cambios en la intensidad de la señal en la secuencia FLAIR indicó que la paciente aún podía tener tejido cerebral potencialmente salvable. La paciente cumplía un criterio de exclusión para la trombólisis intravenosa; por lo tanto, dada la imagen favorable se decidió realizar una trombectomía mecánica. Bajo anestesia general, se punzó la arteria femoral común derecha y se colocó introductor $6.5 \mathrm{~F}$. El arteriograma de la carótida derecha mostró oclusión completa de la M1 derecha ( - Fig. 2A).

Luego se colocó un catéter guía 6F Chaperon (Microvention, Terumo) en la arteria carótida interna derecha. Se hizo avanzar el microcatéter Headaway 27 (Microvention, Terumo) sobre guía Traxcess 14 (Microvention Terumo) hacia el interior de la received June 8, 2017 accepted October 14, 2017 published online April 10, 2018
DOI https://doi.org/ $10.1055 / \mathrm{s}-0038-1639491$ ISSN 1852-9992.
Copyright $\odot$ 2019, Sociedad Argentina de Radiología. Publicado por Thieme Revinter Publicações Ltda., Rio de Janeiro, Brazil. Todos los derechos reservados.
License terms

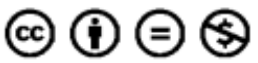



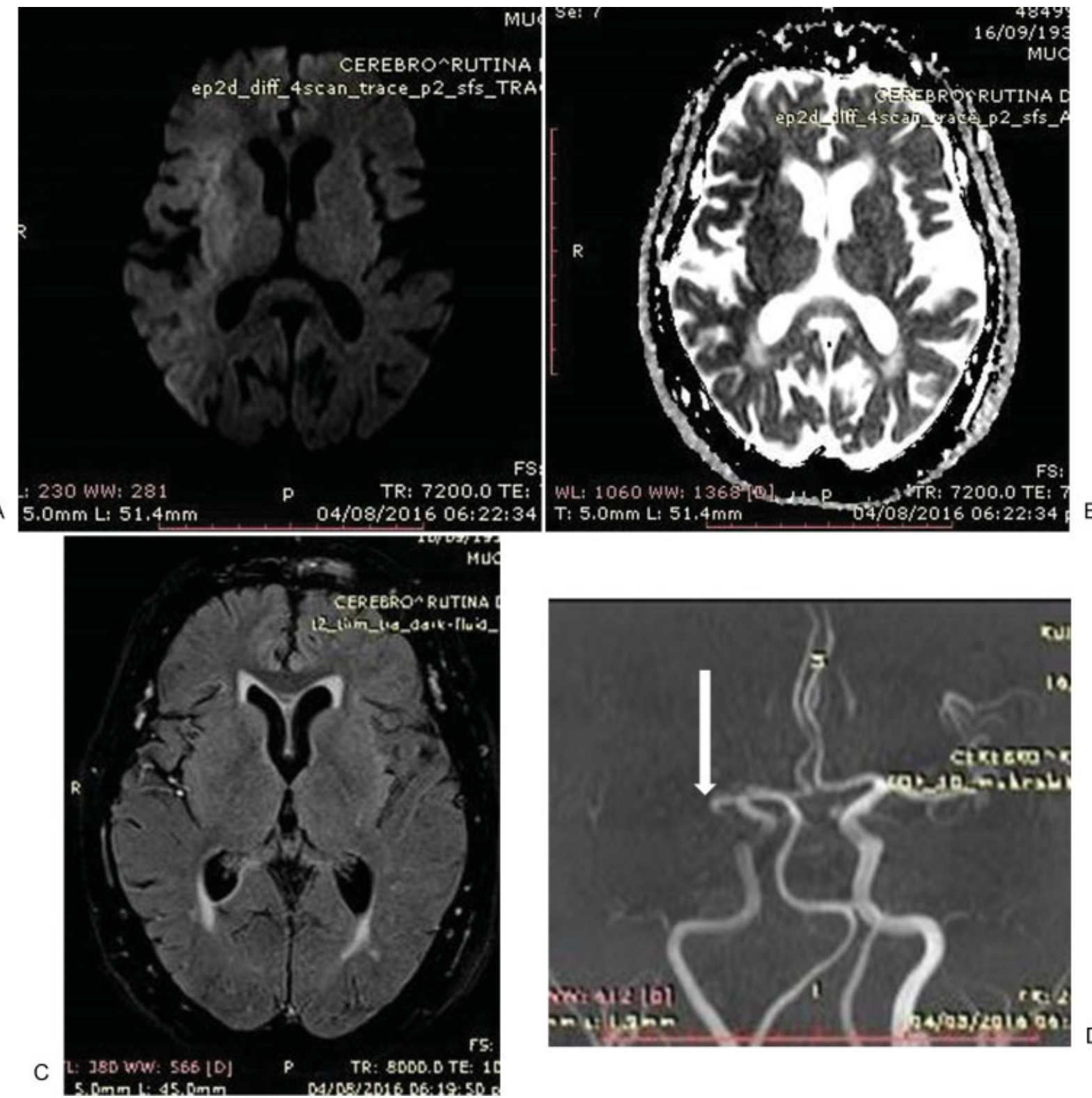

Fig. 1 Antes del tratamiento, la secuencia DWI-ADC mostró restricción en el territorio de la ACM profunda y superficial (A y B) mientras que la secuencia FLAIR fue normal (C); el territorio irrigado por la arteria cerebral anterior no muestra cambios en esas secuencias. La angioRM mostró oclusión de la ACM proximal derecha (D).

arteria M1 ocluida. El primer intento de recuperación se realizó con un dispositivo de revascularización de $4 \mathrm{~mm} \times 20 \mathrm{~mm}$ Solitaire FR (Covidien) que fue desenvainado y se retiró el microcatéter entero mientras se aplicaba aspiración al catéter guía mediante una jeringa; un coágulo se adhirió al stent. Sin embargo, la angiografía de control demostró oclusión permanente sin restauración del flujo. Se realizaron un segundo y un tercer intento con un stent recuperador Solitaire de $4 \times 20 \mathrm{~mm}$ (Covidien); cuando el stent estaba abierto el angiograma mostró flujo a través del stent y de las ramas distales de la arteria cerebral media. Sin embargo, cuando se retiró el dispositivo Solitaire, los angiogramas de control mostraron que la M1 seguía ocluida. En ese momento, decidimos intentarlo una vez más, pero esta vez desplegando el stent Solitaire en la M1. Se realizó la angiografía de control y se observó restablecimiento del flujo. Ese último intento produjo la recanalización completa de la rama con flujo anterógrado normal, TICI 3 (-Fig. 2B). Luego se retiraron los catéteres y se alcanzó hemostasia por compresión manual sobre la punción femoral derecha.

La paciente fue trasladada a la unidad de terapia intensiva para los cuidados posteriores de rutina durante cinco días. Inmediatamente después del procedimiento, la paciente recibió aspirina $325 \mathrm{mg}$ y Clopidogrel $300 \mathrm{mg}$. Al día siguiente, continuó con aspirina $325 \mathrm{mg}$ y Clopidogrel $75 \mathrm{mg}$. Su puntaje en la escala NIHSS a las 72 horas era cero. Se realizó una tomografía computada (TC) sin contraste de seguimiento a las 48 horas, que no mostró infarto ( - Fig. 3), sino una pequeña zona de hemorragia subaracnoidea (HSA) en la convexidad parietal. La paciente fue dada de alta al domicilio sin daños 
A
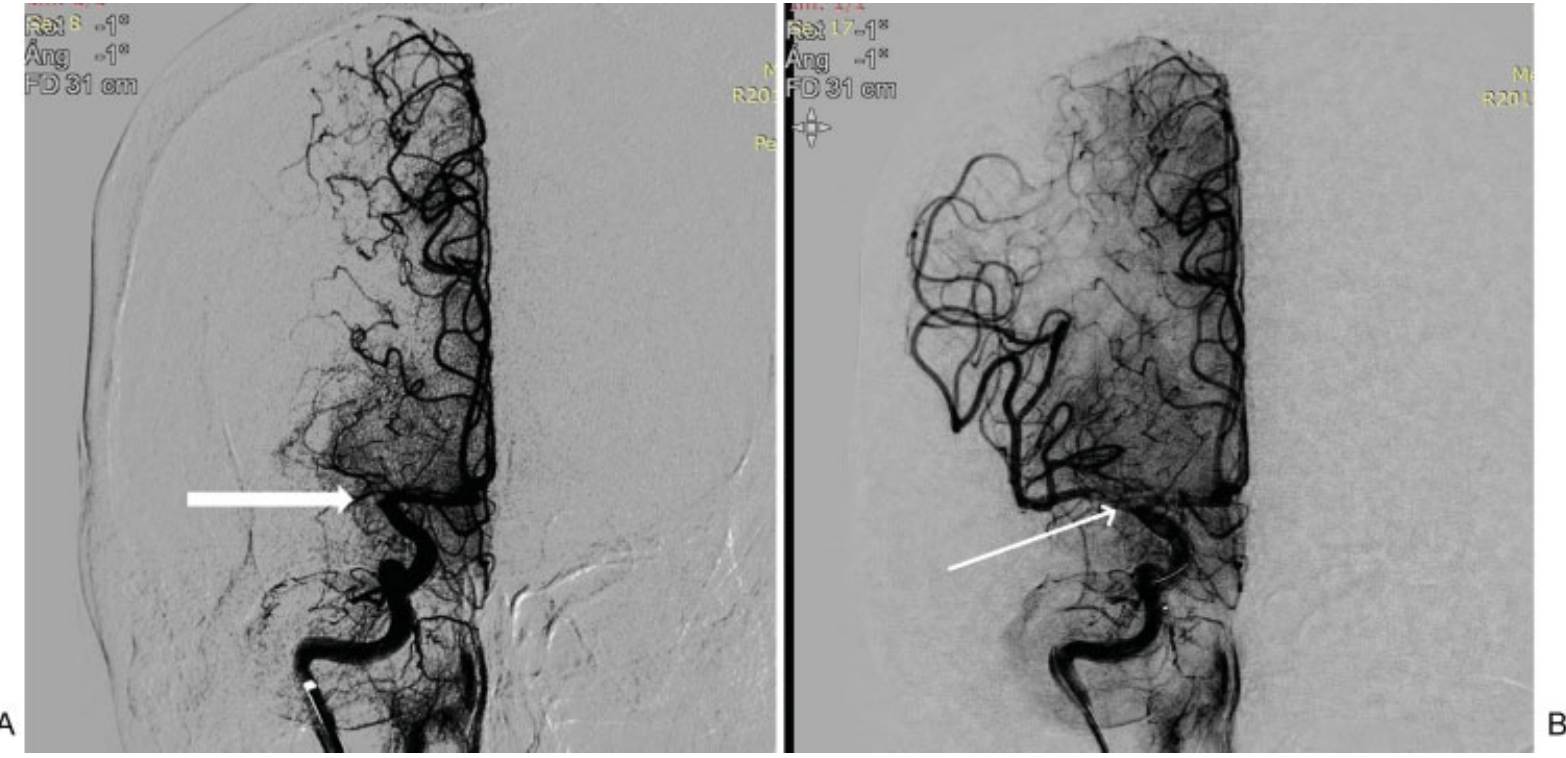

Fig. 2 Proyecciones frontales de la angiografía de la carótida interna derecha. Pretratamiento, se detecta una oclusión a nivel de la ACM-M1 proximal derecha (A). Postratamiento, ese último intento produjo la recanalización completa de la rama con flujo anterógrado normal. Trombólisis en la escala de Infarto Cerebral (TICI) 3 (B).

neurológicos 12 días después del episodio. Dieciocho días después, se realizó una RM de control ( - Fig. 4). El área de leve hiperintensidad en el territorio de la ACM profunda derecha ahora se había normalizado en la secuencia DWI sin disminución del coeficiente de difusión aparente. La secuencia FLAIR ponderada en T2 no mostraba cambios en la intensidad de señal del parénquima en el territorio de la ACM. La angiografía por RM TOF en 3D no mostraba oclusiones de la

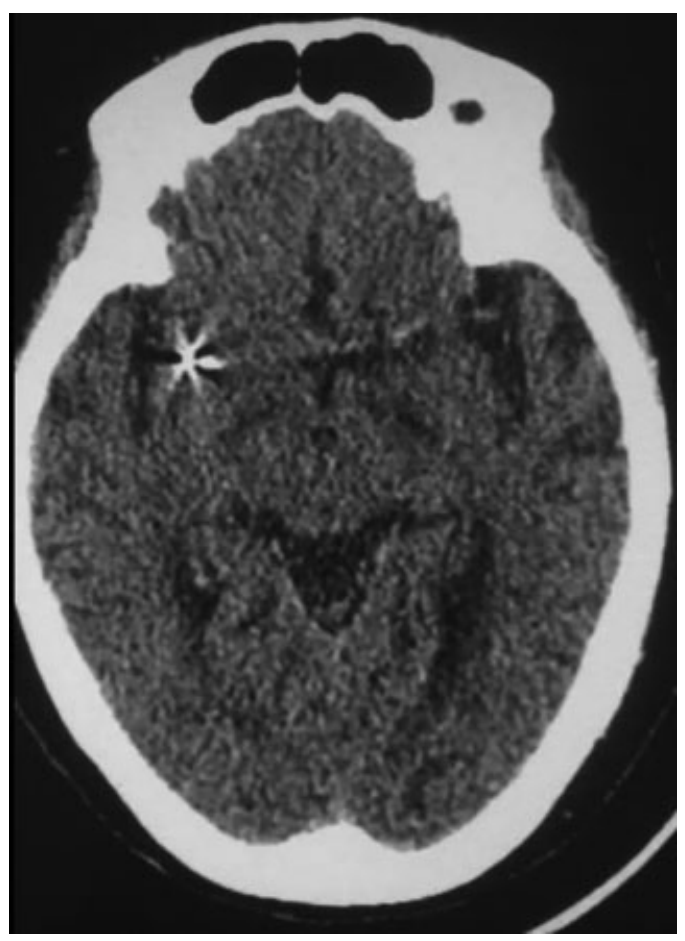

Fig. 3 La TC sin contraste realizada a las 48 horas muestra ausencia de infarto y el stent. arteria. Se observaba una pequeña área de HSA subaguda en la convexidad parietal izquierda.

Los estudios epidemiológicos revelan un patrón circadiano para el accidente cerebrovascular isquémico, con una mayor frecuencia de presentación durante las primeras horas de la mañana y una menor frecuencia durante el sueño. ${ }^{4-6}$ Los estudios por imágenes del cerebro en pacientes con ACV isquémico del despertar indican un posible patrón de ACV tardío durante el sueño. ${ }^{7}$

La ausencia de un momento claro de inicio de los síntomas en el ACV del despertar limita la capacidad de establecer la elegibilidad para las terapias de reperfusión aguda; esa es la razón por la que los estudios diagnósticos de neuroimagen cumplen un papel sumamente importante en esos pacientes. La base de la selección y el tratamiento del ACV del despertar es suplantar "el reloj de tiempo" por el "reloj del tejido."

Las técnicas avanzadas de neuroimagen estiman el volumen de tejido cerebral potencialmente en riesgo de progresión al infarto si no se produce recanalización. La diferencia volumétrica entre el infarto establecido y la penumbra, si está presente, se conoce como mismatch y representa un biomarcador racional para la selección del tratamiento. Se han realizado algunos estudios utilizando DWI e imágenes ponderadas en perfusión (PWI), y otros estudios empleando secuencias DWI y FLAIR de resonancia magnética para identificar tejido infartado en comparación con tejido en riesgo. ${ }^{8,9}$ Las secuencias DWI y FLAIR detectan cambios en el agua cerebral, pero en diferentes secuencias temporales. La secuencia DWI es muy sensible a los cambios tempranos en el agua cerebral; sin embargo, las alteraciones observadas no cambian una vez que aparecen después de los primeros minutos de isquemia, por lo tanto, no se puede determinar el momento exacto de una lesión isquémica con la secuencia DWI. La secuencia FLAIR basada en T2 mide la acumulación de edema cerebral a medida que avanza el proceso de infarto. Por lo tanto, 


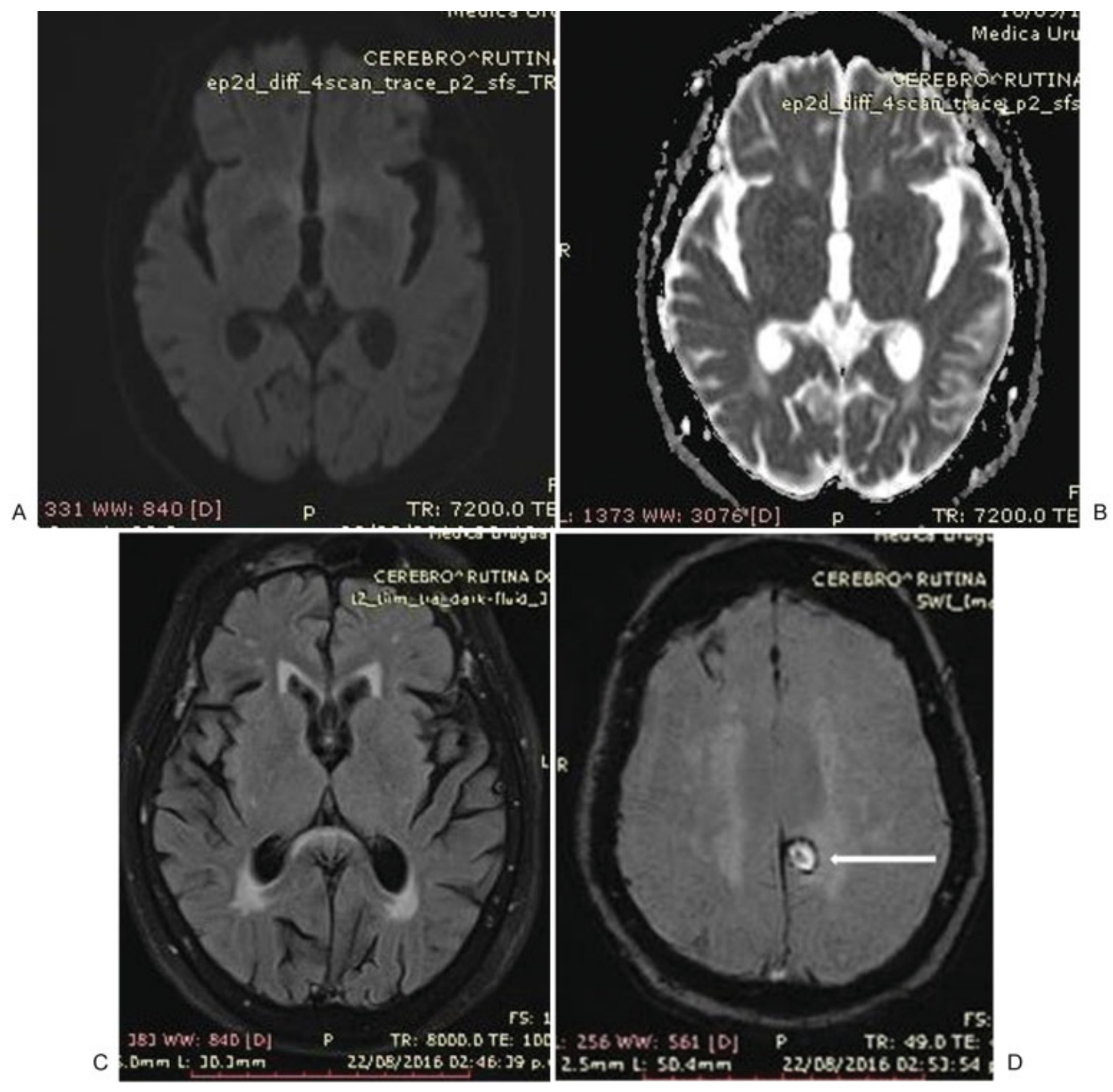

Fig. 418 días después del tratamiento la secuencia DWI-ADC se revirtió en el territorio de la ACM profunda y superficial; siendo normal (A y B), mientras que la secuencia FLAIR también fue normal (C). La secuencia GRE mostró una pequeña zona de HSA subaguda en la convexidad parietal izquierda (D).

en principio, la presencia de una lesión en la secuencia DWI y ausencia de una alteración equivalente en la secuencia FLAIR debería representar un infarto relativamente temprano. Esa idea ha sido estudiada en varios estudios piloto de centro único e investigaciones multicéntricas. ${ }^{3,9}$ En general, se halló que el mismatch DWI-FLAIR identifica con mucha exactitud el tejido isquémico más allá de las 3 a 6 horas y puede identificar isquemia dentro de la ventana de 3 a 4,5 horas con excelente especificidad.

Asimismo, un estudio reciente demostró que una hiperintensidad focal en FLAIR dentro de lesiones agudas en DWI, podría servir como predictor de hemorragia intracerebral sintomática después de la trombólisis. ${ }^{10}$ Por lo tanto, quizás la secuencia FLAIR también pueda ser un buen indicador de una etapa en la que el tratamiento ya no es eficaz o incluso se ha vuelto perjudicial.

Confidencialidad de los datos

Los autores declaran que han seguido los protocolos de su centro de trabajo sobre la publicación de datos de pacientes y que todos los pacientes incluidos en el estudio han recibido información suficiente y han dado su consentimiento informado por escrito para participar en dicho estudio.

Conflicto de Intereses

Los autores del trabajo declaran no tener ningún conflicto de intereses. 


\section{Bibliografía}

1 Mackey J, Kleindorfer D, Sucharew H, et al. Population-based study of wake-up strokes. Neurology 2011;76(19):1662-1667

2 Fink JN, Kumar S, Horkan C, et al. The stroke patient who woke up: clinical and radiological features, including diffusion and perfusion MRI. Stroke 2002;33(04):988-993

3 Thomalla G, Cheng B, Ebinger M, et al; STIR and VISTA Imaging Investigators. DWI-FLAIR mismatch for the identification of patients with acute ischaemic stroke within $4.5 \mathrm{~h}$ of symptom onset (PRE-FLAIR): a multicentre observational study. Lancet Neurol 2011;10(11):978-986

4 Elliott WJ. Circadian variation in the timing of stroke onset: a meta-analysis. Stroke 1998;29(05):992-996

5 Casetta I, Granieri E, Fallica E, la Cecilia O, Paolino E, Manfredini R. Patient demographic and clinical features and circadian variation in onset of ischemic stroke. Arch Neurol 2002;59 (01):48-53
6 Marler JR, Price TR, Clark GL, et al. Morning increase in onset of ischemic stroke. Stroke 1989;20(04):473-476

7 Huisa BN, Raman R, Ernstrom K, et al. Alberta Stroke Program Early CT Score (ASPECTS) in patients with wake-up stroke.J Stroke Cerebrovasc Dis 2010;19(06):475-479

8 Huisa BN, Liebeskind DS, Raman R, et al; University of California, Los Angeles Stroke Investigators. Diffusion-weighted imaging-fluid attenuated inversion recovery mismatch in nocturnal stroke patients with unknown time of onset. J Stroke Cerebrovasc Dis 2013;22(07):972-977

9 Thomalla G, Rossbach P, Rosenkranz M, et al. Negative fluidattenuated inversion recovery imaging identifies acute ischemic stroke at 3 hours or less. Ann Neurol 2009;65(06):724-732

10 Cho AH, Kim JS, Kim SJ, et al. Focal fluid-attenuated inversion recovery hyperintensity within acute diffusion-weighted imaging lesions is associated with symptomatic intracerebral hemorrhage after thrombolysis. Stroke 2008;39(12):3424-3426 